\title{
Expression of the promyelocytic leukemia protein without the nuclear localization signal as a novel diagnostic marker for acute promyelocytic leukemia
}

\author{
TING XU ${ }^{1,2^{*}}$, XIAO-QUN YANG ${ }^{1,2^{*}}$, KAI-LING JIANG ${ }^{2}$, HUI WANG ${ }^{2}$, PENG-PENG MA ${ }^{2}$, \\ LIANG ZHONG ${ }^{2}$ and BEI-ZHONG LIU ${ }^{1,2}$ \\ ${ }^{1}$ Central Laboratory of Yong-Chuan Hospital, Chongqing Medical University; ${ }^{2}$ Key Laboratory of \\ Laboratory Medical Diagnostics, Ministry of Education, Department of Laboratory Medicine, \\ Chongqing Medical University, Chongqing 400016, P.R. China
}

Received May 19, 2016; Accepted June 29, 2016

DOI: $10.3892 /$ or.2017.5357

\begin{abstract}
Promyelocytic leukemia-retinoic acid receptor $\alpha$ (PML-RAR $\alpha$ ) is a fusion protein generated by the $t(15 ; 17)$ (q22;q12) translocation associated with acute promyelocytic leukemia (APL). PML-RAR $\alpha$ is cleaved by neutrophil elastase, an early myeloid-specific serine protease, leading to translocation of the nuclear localization signal (NLS) of the PML protein to the N-terminal of RAR $\alpha$, and the mutational product PML(NLS ${ }^{-}$). The present study was designed to analyze the role of the NLS in mediating PML transport into the nucleus and to evaluate the value of measuring NLS translocation in the early diagnosis of APL. PML and PML(NLS ${ }^{-}$) localization was examined by immunofluorescence (IF). The interaction between PML/PML(NLS ${ }^{-}$) and importin $\alpha$ was detected by an in vivo binding assay using co-immunoprecipitation and double IF labeling. Twenty-seven untreated APL patients with PML-RAR $\alpha$ and 22 non-APL controls were evaluated. PML(NLS ${ }^{-}$) was detected in primary APL, but not non-APL cells. IF showed that PML was localized to the nucleus, interacted with importin $\alpha$ in vivo, and co-localized in the PML nuclear bodies. PML(NLS $\left.{ }^{-}\right)$was primarily localized in the cytoplasm and the interaction with importin $\alpha$ was lost. IF had
\end{abstract}

Correspondence to: Professor Bei-Zhong Liu, Central Laboratory of Yong-Chuan Hospital, Chongqing Medical University, 1 Yixueyuan Road, Chongqing 400016, P.R. China

E-mail: liubeizhong@cqmu.edu.cn

Dr Liang Zhong, Key Laboratory of Laboratory Medical Diagnostics, Ministry of Education, Department of Laboratory Medicine, Chongqing Medical University, 1 Yixueyuan Road, Chongqing 400016, P.R. China

E-mail: cnmed1@hotmail.com

${ }^{*}$ Contributed equally

Key words: acute promyelocytic leukemia, biomarker, clinical diagnosis, nuclear localization signal, promyelocytic leukemia a sensitivity and specificity of 92.6 and $77.3 \%$, respectively, for diagnosing APL. These data suggest that PML(NLS ${ }^{-}$) may be a novel diagnostic biomarker for APL.

\section{Introduction}

Acute promyelocytic leukemia (APL) is a distinct subtype of acute myeloid leukemia (AML). APL is characterized by the selective expansion of immature myeloid precursor cells that are blocked at the promyelocytic stage. Almost $98 \%$ of patients with APL have transformed promyelocytes carrying the $t(15 ; 17)(q 22 ; q 12)$ translocation that generates the promyelocytic leukemia-retinoic acid receptor $\alpha$ (PML-RAR $\alpha)$ fusion gene product (1). PML-RAR $\alpha$ has been proposed to act as a dominant negative inhibitor of endogenous PML and RAR $\alpha$ functions, causing resistance to apoptosis and blocking myeloid differentiation (2).

Several studies revealed that transgenic and knock-in animal models expressing PML-RAR $\alpha$ in early myeloid cells developed APL (3-5). However, when PML-RAR $\alpha$ was expressed in late myeloid cells, APL did not develop (6). Moreover, one study suggested that PML-RAR $\alpha$ may require cleavage to become fully oncogenic (7).

Neutrophil elastase (NE), an early myeloid-specific serine protease, cleaves the PML-RAR $\alpha$ protein of bcr-1 into two parts. Mice deficient in NE develop APL at a greatly reduced rate when crossed with PML-RAR $\alpha$ knock-in mice (8). We recently found that overexpression of the PML protein lacking the nuclear localization signal [PML(NLS $\left.{ }^{-}\right)$] can inhibit apoptosis and promote the growth of HL60 cells (9). We also found that NLS-RAR $\alpha$ promotes proliferation and inhibits differentiation of HL60 cells (10). Together, these observations suggest that activation of PML-RAR $\alpha$ by NE-mediated cleavage plays a key role in the pathogenesis of APL.

PML is a RING finger protein that belongs to the tripartite motif protein family (11). There are several variants of human PML generated by alternative splicing with variable C-terminal lengths, and sizes ranging from 47 to $160 \mathrm{kDa}$ (12). However, all isoforms have the same N-terminus containing the NLS, B-Boxes, and an $\alpha$-helical coiled region. PML is an essential 
component of highly dynamic nuclear structures known as PML oncogenic domains or nuclear bodies (NBs) (13). PML and PML NBs have been implicated in regulating growth inhibition, senescence and apoptosis (14), and a myriad of PML activities have been linked to its function in NB formation. Deregulation of PML can be oncogenic and has been observed in multiple human cancers (15). PML can be SUMOylated on three different lysine residues and contains a SIM domain. Lys65 in the RING finger domain, Lys160 in the B1 Box, and Lys490 in the NLS contribute three major sentrinization sites (16-18).

In normal human cells, PML has a nuclear localization. A minority of PML types (such as PML VIb) show a cytoplasmic localization (19). The best understood system for the transport of macromolecules between the cytoplasm and the nucleus is the classical nuclear import pathway. In this pathway, a protein containing a basic NLS is imported by a heterodimeric receptor consisting of $\beta$-karyopherin. This receptor mediates interactions between the nuclear pore complex and the adaptor protein importin $\alpha$, which binds directly to the classical NLS (20). This indicates that the NLS sequence is critical for both PML SUMOylation and PML NB formation, and that a deficiency of the NLS sequence influences PML nuclear localization.

In the present study, PML and PML(NLS $\left.{ }^{-}\right)$expression was investigated in normal human cells and APL blasts. An immunofluorescence co-localization assay and co-immunoprecipitation were used to demonstrate that PML was localized in the nucleus by its NLS sequence interacting with importin $\alpha$. However, aberrant PML(NLS ${ }^{-}$) disrupted the activity of the NLS in the cytoplasm and eliminated the interaction with importin $\alpha$. These results indicated that PML(NLS ${ }^{-}$) may be a potential biomarker for APL.

\section{Materials and methods}

Cell lines and primary APL blasts. HL-60, NB4 and HEK293 cell lines were purchased from the Shanghai Institutes for Biological Sciences (Shanghai, China). HEK293 cells were grown at $37^{\circ} \mathrm{C}$ with $5 \% \mathrm{CO}_{2}$ in Dulbecco's modified Eagle's medium supplemented with $10 \%$ fetal bovine serum (FBS; Gibco, Grand Island, NY, USA) and $5 \mathrm{mg} / \mathrm{ml}$ of gentamicin (Invitrogen, Carlsbad, CA, USA). HL-60 and NB4 cells were cultured in RPMI-1640 (Gibco) supplemented with 10\% FBS, $100 \mathrm{U} / \mathrm{ml}$ penicillin and $100 \mu \mathrm{g} / \mathrm{ml}$ streptomycin.

APL blast cells were obtained from human bone marrow aspirates. Leukemia cells were isolated using Ficoll-Hypaque (Sigma-Aldrich, St. Louis, MO, USA) density gradient centrifugation.

Patient samples. Bone marrow and peripheral blood samples were obtained with informed consent, in accordance with the Declaration of Helsinki, from new untreated APL patients or patients with non-APL hematological disorders (the controls), presenting at the Department of Hematology, The First Affiliated Hospital of Chongqing Medical University. Blasts were $>70 \%$ in all APL samples. Hemograms, peripheral blood smears, and bone marrow examinations were performed in all APL and control cases using Jenner-Giemsa and cytochemistry for routine diagnostic morphology. The results were compared
Table I. Clinical details of patients.

\begin{tabular}{lccccr}
\hline $\begin{array}{l}\text { Patient } \\
\text { no. }\end{array}$ & Gender & $\begin{array}{c}\text { Age } \\
(\text { years })\end{array}$ & FAB & $\begin{array}{c}\text { Blast } \\
(\%)\end{array}$ & $\begin{array}{r}\text { WBC } \\
\left(\times 10^{9}\right)\end{array}$ \\
\hline 001 & F & 16 & M3 & 85 & 30.5 \\
002 & F & 25 & M3 & 79 & 3.2 \\
003 & M & 30 & M3 & 72 & 2.5 \\
004 & M & 29 & M2 & 75 & 17.8 \\
005 & F & 33 & M2 & 70 & 22.9 \\
006 & M & 22 & M4 & 66 & 10.5 \\
007 & M & 35 & M4 & 69 & 25.7 \\
\hline
\end{tabular}

F, female; M, male; FAB, acute myeloid leukemia subtype, according to the French-American-British classification; WBC, white blood cell count.

with those obtained by immunofluorescence staining. Details of the patients examined in the present study are presented in Table I.

Analyses of transfected cells. PML and PML(NLS-) eukaryotic plasmids encoding C-terminal HA-tagged proteins [PCMV-HA-PML and PCMV-HA-PML(NLS ${ }^{-}$), respectively], and the importin $\alpha$ expression vector encoding C-terminal Myc-tagged proteins (PCMV-MYC-imp $\alpha$ ), were constructed for indirect immunofluorescence and the in vivo binding assay. Briefly, HEK293 cells seeded on $75 \mathrm{~cm}^{2}$ dishes or plated on coverslips, were transfected with tagged constructs using Lipofectamine (Invitrogen) according to the manufacturer's instructions. Western blotting and immunofluorescence localization assays were performed by conventional methods to investigate the expression of PML and PML(NLS $\left.{ }^{-}\right)$. Co-immunoprecipitation was used to identify the interaction of PML/PML(NLS $\left.{ }^{-}\right)$with importin $\alpha$. NB4 cells $\left(2 \times 10^{6}\right)$ were electroporated with $7 \mu \mathrm{g}$ of supercoiled plasmid DNA containing the NE cDNA pcDNA3.1. Cells were harvested at various time points after electroporation, and cell lysates were prepared for co-immunoprecipitation.

Western blotting. Cells were washed with ice-cold phosphate-buffered saline (PBS) and lysed in cell lysis buffer [0.5\% Triton $\mathrm{X}-100,150 \mathrm{mM} \mathrm{NaCl}, 20 \mathrm{mM}$ Tris ( $\mathrm{pH} 7.5)$, $5 \mathrm{mM}$ EDTA and $1 \mathrm{mM}$ phenylmethanesulfonyl fluoride] on ice for $20 \mathrm{~min}$. Lysates were pelleted by centrifugation at $14,000 \mathrm{x} \mathrm{g}$ for $10 \mathrm{~min}$ at $4^{\circ} \mathrm{C}$. Total protein concentrations in the supernatant were determined using a Bio-Rad protein assay (Bio-Rad, Hercules, CA, USA). Whole cell lysates were separated by $10 \%$ sodium dodecyl sulfate-polyacrylamide gel electrophoresis and transferred to polyvinylidene difluoride membranes (Millipore, Darmstadt, Germany). Membranes were blocked with 5\% milk in PBS for $1 \mathrm{~h}$ and incubated with primary antibodies at $4^{\circ} \mathrm{C}$ overnight. A polyclonal rabbit anti-PML antibody (ab72137; Abcam, Cambridge, UK) and a polyclonal rabbit anti-actin antibody (Santa Cruz Biotechnology, Santa Cruz, CA, USA) were used as primary antibodies. Immunocomplexes were detected with appropriate horseradish peroxidase-conjugated secondary antibodies and 


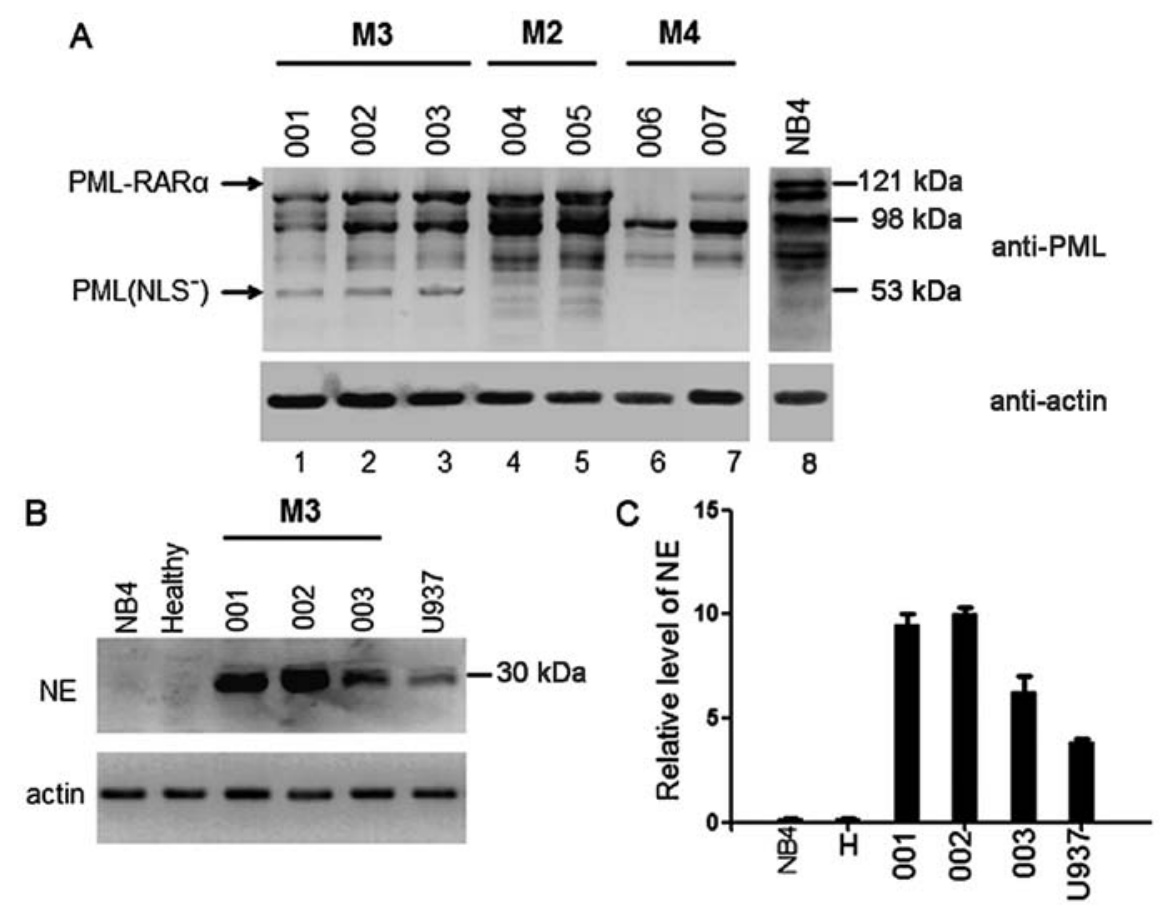

Figure 1. PML-RAR $\alpha$ cleavage activity in primary human M3 AML cells. (A) Western blot analyses for PML and actin in protein extracts from primary bone marrow AML cells. Leukemic bone marrow cells from patients 001 (lane 1), 002 (lane 2) and 003 (lane 3) harbor a bcr-1 PML-RAR $\alpha$ mRNA. Lane 8 presents NB4 cells as a control. (B) Western blot analyses for NE in protein extracts from peripheral blood neutrophils of APL patients. Lane 1 presents NB4 cells as a negative control. Lane 6 presents U937 cells as a positive control. (C) In vitro expression levels of NE in extracts derived from the indicated cell lines and primary human APL cells. Data are presented as means \pm SD of each lysate and the analysis was repeated three times.

detected by enhanced chemiluminescence (GE Healthcare, Marlborough, MA, USA).

Immunofluorescence microscopy. Transfected or untransfected HEK293 cells were cultured on glass slides and fixed in $4 \%$ paraformaldehyde in PBS at $4^{\circ} \mathrm{C}$ for $20 \mathrm{~min}$. Cytospins of non-adherent cells were performed with 40,000 cells at $400 \mathrm{rpm}$ for $10 \mathrm{~min}$, followed by air-drying and fixation as previously described (21). After blocking with $1 \%$ bovine serum albumin for $30 \mathrm{~min}$, slides were incubated for $1 \mathrm{~h}$ at room temperature with a rabbit polyclonal anti-PML antibody (Abcam) or a mouse monoclonal anti-importin $\alpha$ antibody (Sigma-Aldrich), diluted at 1:200 in Tris-buffered saline with Tween-20 (TBST). Fluorescein isothiocyanate-conjugated goat anti-rabbit IgG and tetramethylrhodamine isothiocyanate-conjugated goat anti-mouse IgG (both from CW Biotech, Beijing, China) were used as the secondary antibodies. Cells were mounted in mounting media (Sigma-Aldrich) containing $1 \mu \mathrm{g} / \mathrm{ml}$ 4',6-diamidino-2-phenylindole (DAPI) (Beyotime, Shanghai, China) and visualized by standard immunofluorescence microscopy (Olympus BX51; Olympus, Tokyo, Japan).

Co-immunoprecipitation. For in vivo binding assays, cells were seeded in $75 \mathrm{~cm}^{2}$ plates. Collected cells were lysed in ice-cold cell lysis buffer $(20 \mathrm{mM}$ Tris, $150 \mathrm{mM} \mathrm{NaCl}, 1 \mathrm{mM}$ EDTA, $100 \mu \mathrm{g} / \mathrm{ml}$ phenylmethanesulfonyl fluoride and $1 \%$ NP40). One milligram of protein was incubated with an anti-PML or anti-importin $\alpha$ antibody at $4^{\circ} \mathrm{C}$ overnight. Normal mouse or rabbit IgG (Santa Cruz Biotechnology) was used as a negative control. Protein A/G-Sepharose beads were added for $5 \mathrm{~h}$ at $4^{\circ} \mathrm{C}$ to bind the immune complex from the incubation solution. Precipitates were washed four times with lysis buffer, dissociated by boiling with the sample buffer, and subjected to sodium dodecyl sulfate-polyacrylamide gel electrophoresis. The remaining immunoblotting procedures are described in the western blotting section in the 'Materials and methods'. Co-immunoprecipitated proteins were visualized using anti-PML, anti-importin $\alpha$, anti-HA tag or anti-cMyc tag antibodies (Abcam).

\section{Results}

APL cells derived from human M3 patients contain the mutant $P M L\left(N L S^{-}\right)$protein. To verify whether PML-RAR $\alpha$ was cleaved in vivo, primary leukemia cells were obtained from the bone marrow of three patients with M3 AML (APL), two with M2, and two with M4 as negative controls. APL cells from the M3 patients harbored a bcr-1 PML-RAR $\alpha$ fusion mRNA, which predicts a protein of $120 \mathrm{kDa}$. Western blotting performed on lysates of primary AML cells showed that none of the M3 samples expressed the full-length $120 \mathrm{kDa}$ PML-RAR $\alpha$ protein. Instead, each contained multiple protein fragments recognized by the anti-PML antibody, and a mutant PML protein of $\sim 53 \mathrm{kDa}$ (Fig. 1A, lanes 1-3). Samples derived from M2 and M4 AML cells contained no detectable PML-RAR $\alpha$ protein as expected, although multiple proteins were recognized by the anti-PML antibody (Fig. 1A, lanes 4-7).

NB 4 cells that contained the bcr-1 isoform of PML-RAR $\alpha(22,23)$ expressed the predicted $120 \mathrm{kDa}$ PML-RAR $\alpha$ protein (Fig. 1A, lane 8), but did not express NE. Samples derived from healthy cells also contained no detectable NE protein (Fig. 1B). Western blotting performed 
A

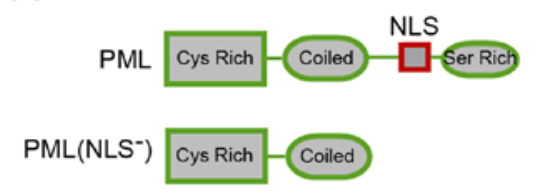

C
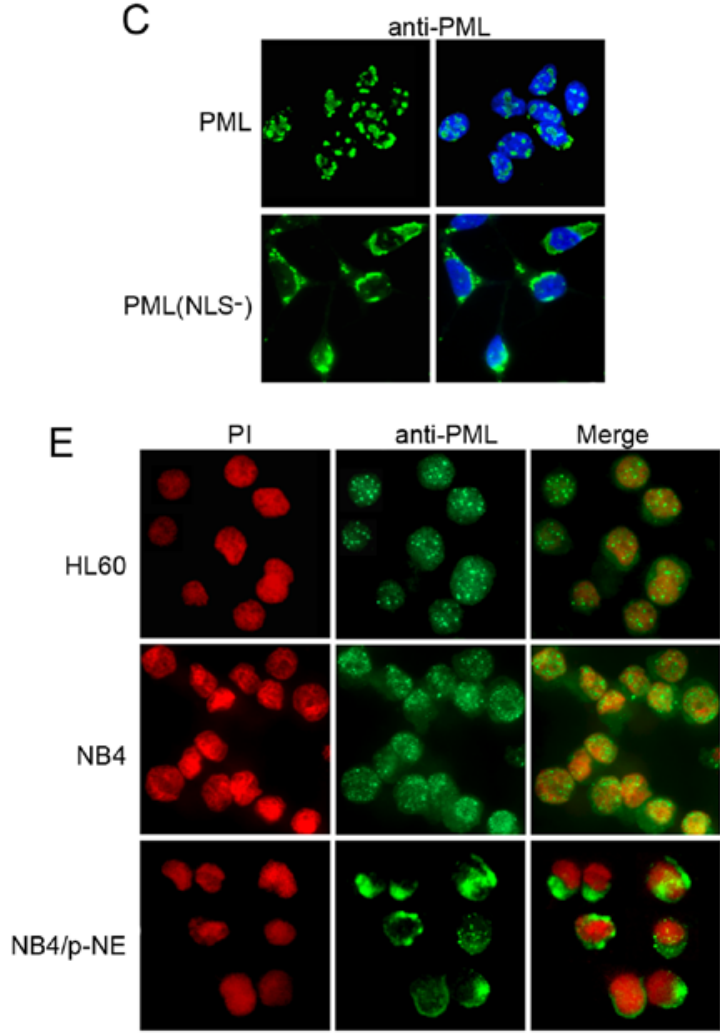

B
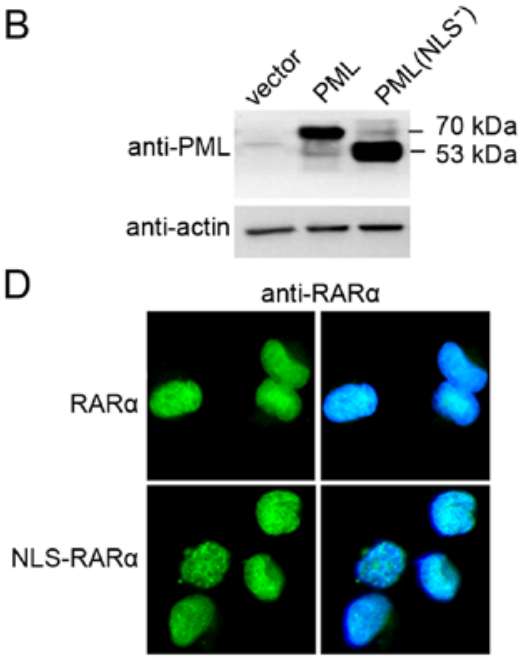

F

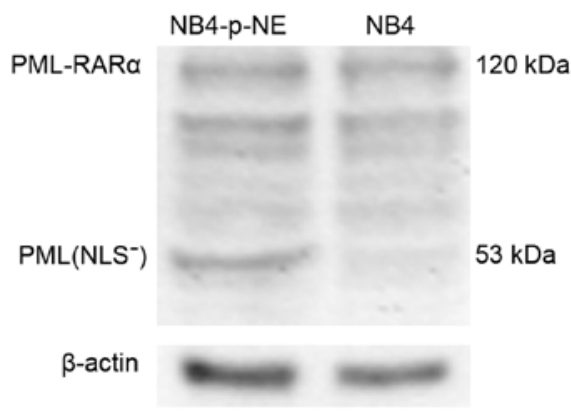

Figure 2. Analyses of PML localization in transfected HEK293 cells and leukemia cell lines. (A) Schematic illustration of PML and its deletion mutant, PML(NLS-). The positions of the NLS and its main domains are indicated. (B) Western blot analysis of transfected HEK293 cells for PML. Vector, pCMV-HA vector transfected control; PML, PML expression vector; PML(NLS), PML(NLS-) expression vector. (C and D) Immunofluorescence analyses of PML, PML(NLS), RAR $\alpha$ and NLS-RAR $\alpha$ in transfected HEK293 cells. HEK293 cells were transfected with the PML, PML(NLS ), RAR $\alpha$ and NLS-RAR $\alpha$ expression vectors. Immunofluorescence staining (green) was performed using anti-PML polyclonal and anti-RAR $\alpha$ polyclonal antibodies, followed by a fluorescein isothiocyanate-conjugated secondary antibody. Nuclei were visualized by DAPI staining. (E) Immunofluorescence microscopy of HL60, NB4 and NB4/p-NE cells $24 \mathrm{~h}$ after electroporation with the NE expression construct. All cells were stained with an anti-PML antibody (green fluorescence). Nuclei were stained with propidium iodide (red fluorescence). (F) Western blot analysis for PML and actin in protein extracts from NB4 and NB4/p-NE cells.

on all three peripheral blood neutrophil extracts showed that, similar to U937 cells, M3 cells expressed the $30 \mathrm{kDa}$ NE protein (Fig. 1B). The quantity of NE protein in the APL samples was much higher than that in the U937 cells (Fig. 1C). In contrast, extracts from healthy and NB4 cells did not contain measurable quantities of NE protein (Fig. 1C).

The NLS is important for the nuclear localization of PML. To determine whether the absence of the NLS sequence influenced the localization of PML, immunofluorescence microscopy was used to determine the subcellular localization of wild-type PML and PML(NLS ${ }^{-}$) (Fig. 2A and C). Transfected cells were easily identified due to the intense signals created by both overexpressed proteins. In western blot analyses of transfected HEK293 cells, the HA-tagged PML or PML(NLS ${ }^{-}$) proteins appeared as a group of bands at 70 and $53 \mathrm{kDa}$, respectively (Fig. 2B). This was consistent with their calculated weights. When wild-type PML was transfected transiently into HEK293 cells, the PML protein was detected as a few large nuclear dots (Fig. 2C). When PML(NLS') was transfected into HEK293 cells, it was detected as large cytoplasmic aggregates (Fig. 2C). This pattern was consistent with the reported cytoplasmic aggregation of full-length PML lacking its NLS (24). When cells were transfected with NLS-RAR $\alpha$ (containing the PML NLS plus the RAR $\alpha$ portion of PML-RAR $\alpha$ ), this protein was detected in a homogeneous nuclear pattern, similar to that of RAR $\alpha$ in RAR $\alpha$-transfected cells (Fig. 2D).

The intracellular expression of PML and PML-RAR $\alpha$ was also examined in APL cell lines. HL60, a human myeloid cell line from peripheral blood leukocytes of a patient with APL (25), does not harbor $\mathrm{t}(15 ; 17)$ and expresses only normal PML. The APL-derived cell line, NB4, is a valuable system to study the expression of PML-RAR $\alpha$ since both the normal and rearranged PML genes are present. Immunofluorescence microscopy of HL60 cells revealed a punctate nuclear pattern of PML expression (Fig. 2E). In NB4 cells, a micro-punctate pattern consisting of hundreds of very small dots, predominately 
A

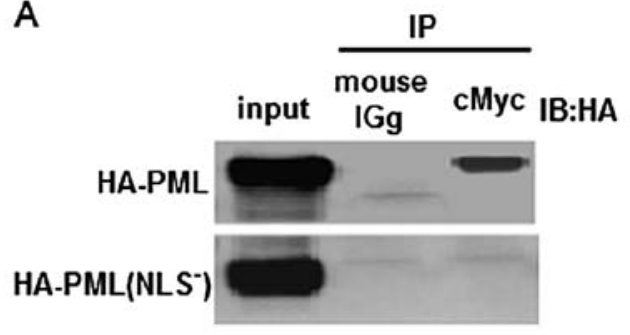

C

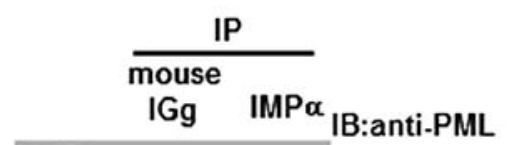

B

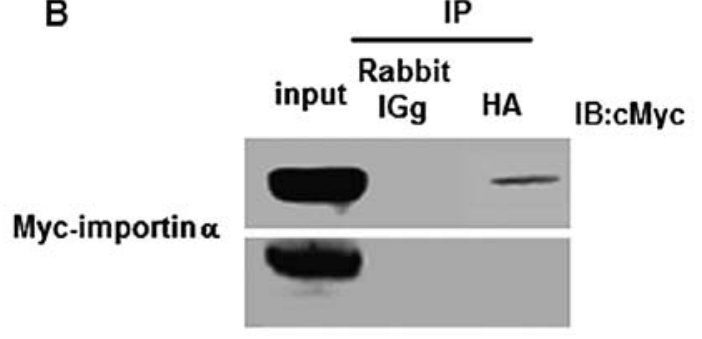

D

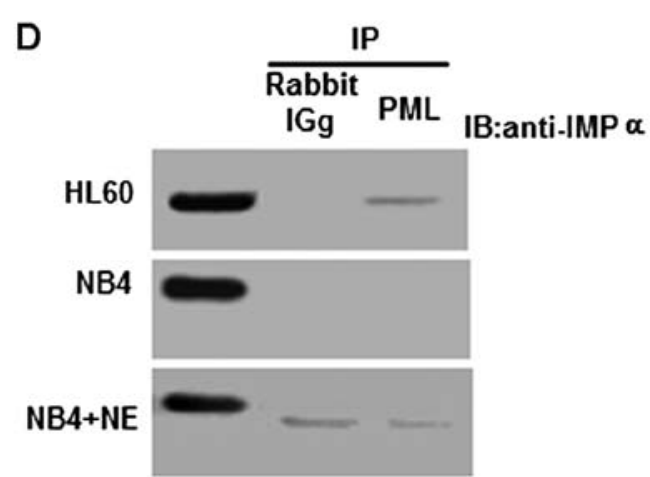

Figure 3. Analyses of the interaction between PML and importin $\alpha$ in transfected HEK293 cells and leukemia cell lines. (A and B) Co-immunoprecipitation was employed to detect the interaction between PML/PML(NLS) and importin $\alpha$ in transfected HEK293 cells. Upper blot, co-transfection with the PML and importin $\alpha$ expression vectors. Lower blot, co-transfection with the PML(NLS) and importin $\alpha$ expression vectors. (A) IP, Anti-cMyc antibody; IB, anti-HA antibody mouse IgG was used as the negative control. (B) IP, Anti-HA antibody; IB, anti-cMyc antibody. Rabbit IgG was used as the negative control. (C and D) Reciprocal immunoprecipitation analyses were used to evaluate the physical interaction between PML and importin $\alpha$ in HL60, NB4 and NB4/-NE cell lines. (C) IP, Anti-importin $\alpha$ antibody; IB, anti-PML antibody. Mouse IgG was used as the negative control. (D) IP, Anti-PML antibody; IB, anti-importin $\alpha$ antibody. Rabbit IgG was used as the negative control.

located in the nucleus, was observed (Fig. 2E). This demonstrated the expected nuclear location of PML-RAR $\alpha$. In contrast, when NB4 cells were transfected with the NE expression vector, the PML-RAR $\alpha$ pattern was almost lost and replaced with a typical PML(NLS) profile as previously shown in NE transfected HEK293 cells. In NB4 cells, at 24 h post-transfection with NE, immunofluorescence microscopy showed that the PML-relevant protein was mainly cytoplasmic, although some nuclear dots were also detected (Fig. 2E). Likewise, western blot analysis revealed that PML(NLS) existed in the NE-transfected cells but not in the parental NB4 cells (Fig. 2F).

$P M L$ and importin $\alpha$ interact in vivo and co-localize in the PML NBs. Our previous study revealed that PML was located in the nucleus and that the NLS was responsible for efficient nuclear targeting. In the present study, HEK293 cells were co-transfected with either PCMV-HA-PML and PCMV-Myc-importin $\alpha$, or PCMV-HA-PML(NLS) and PCMV-Myc-importin $\alpha$. HA-PML, but not ML(NLS) was, co-immunoprecipitated with Myc-importin $\alpha$ (Fig. 3A). When an anti-HA antibody was used to immunoprecipitate HA-PML and HA-PML(NLS), importin $\alpha$ was detected using an anti-Myc antibody. Nonetheless, we were unable to detect an interaction between the HA-PML(NLS) protein and Myc-importin $\alpha$ (Fig. 3B). This demonstrated that the NLS of PML was required for the binding of PML to importin $\alpha$.

To examine whether the same NLS alteration could abrogate the interaction between PML and importin $\alpha$ in NB4 cells, the NE expression vector was electroporated into NB4 cells for an in vivo binding assay. Importantly, a specific interaction was observed between endogenous PML and importin $\alpha$ in HL60 cells, but not in NB4 and NB4/p-NE cells (Fig. 3C and D). These results clearly demonstrated that PML and importin $\alpha$ interacted in vivo at physiological levels, and that there was insufficient PML to detect an interaction with importin $\alpha$ in NB4 and NB4/p-NE cells.

HEK293 cells, co-transfected with HA-PML and Myc-importin $\alpha$, were fixed $24 \mathrm{~h}$ later and subjected to immunofluorescence staining. Importin $\alpha$ co-localized with PML in the PML NBs, although staining of importin $\alpha$ was also diffusely found within the nucleus and was evident in the cytoplasm (Fig. 4A). In contrast, PML(NLS) was mainly localized in the cytoplasm, without co-localization with importin $\alpha$ in the nucleus (Fig. 4A). To verify that co-localization with importin $\alpha$ was not due to the overexpression of PML, the experiment was repeated in HL60 and NB4 cells to determine whether co-localization also occurred at physiological levels of PML. As shown in Fig. 4C, importin $\alpha$ co-localized with endogenous PML in HL60 cells, but not in NB4 cells.

We also examined whether an interaction existed between NLS-RAR $\alpha$ and importin $\alpha$. Based on the immunofluorescence assay, NLS-RAR $\alpha$ was localized in the nucleus but not co-localized with importin $\alpha$. There was no noticeable difference in the localization of NLS-RAR $\alpha$ and RAR $\alpha$, and there was no determinable interaction between NLS-RAR $\alpha$ and importin $\alpha$ or RAR $\alpha$ (Fig. 4B).

Evaluation of $P M L$ for the diagnosis of APL. Although immunofluorescence of NB4 cells transfected with NE demonstrated the expected 'dispersed' cytoplasmic pattern with PML-specific antibodies, the localization of PML and its interaction with importin $\alpha$ were unknown in primary 
A
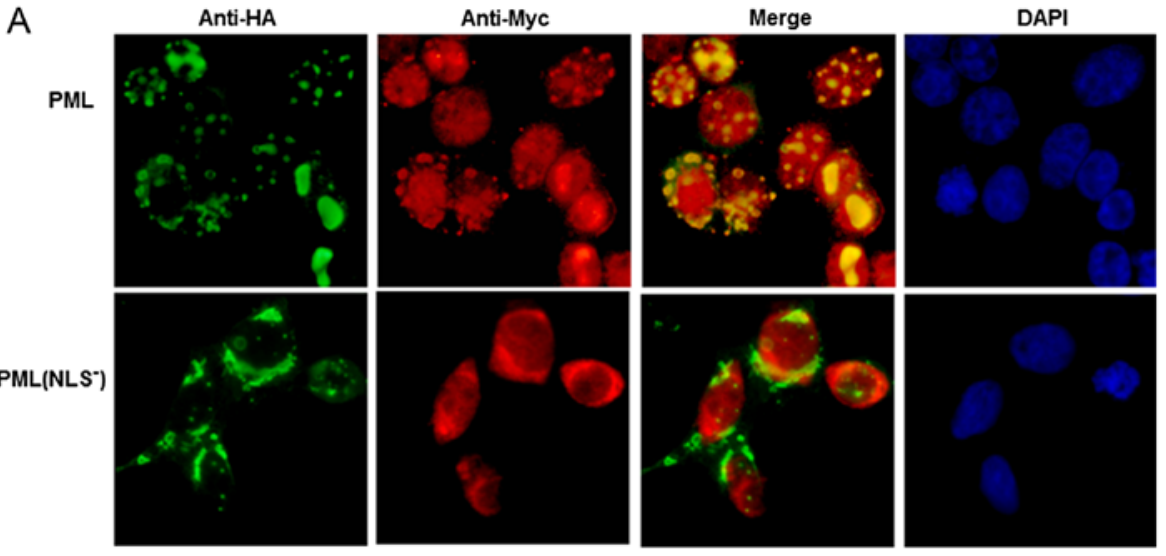

B

Anti-HA
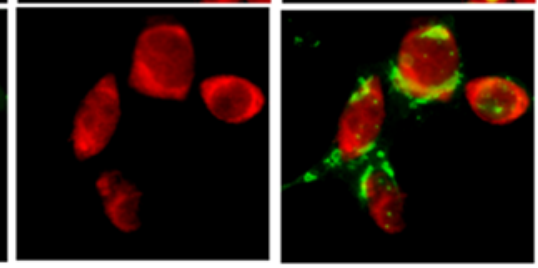

Anti-Myc
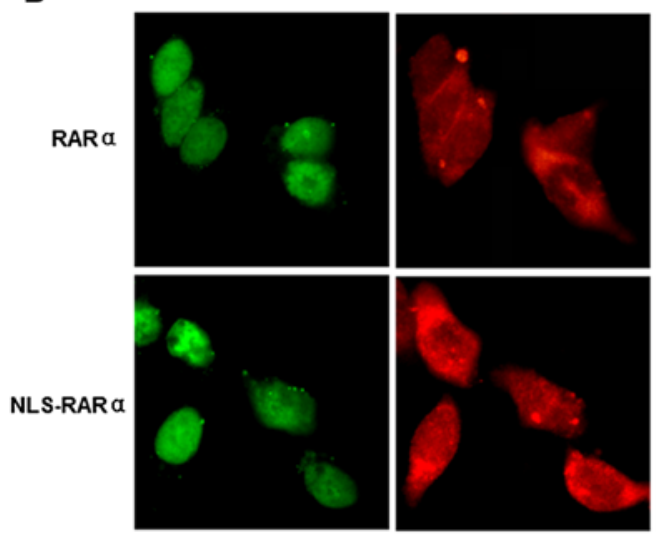

Merge

DAPI
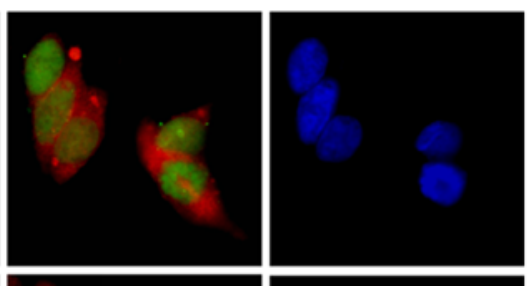

C

\begin{abstract}
FITC/Anti-PML
\end{abstract}
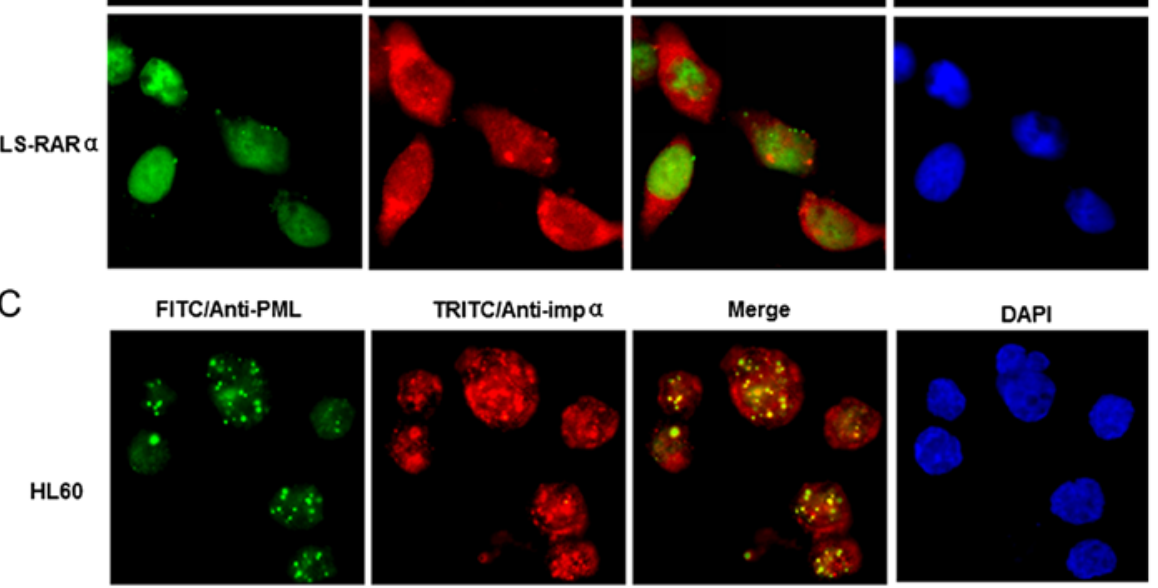

TRITC/Anti-impa
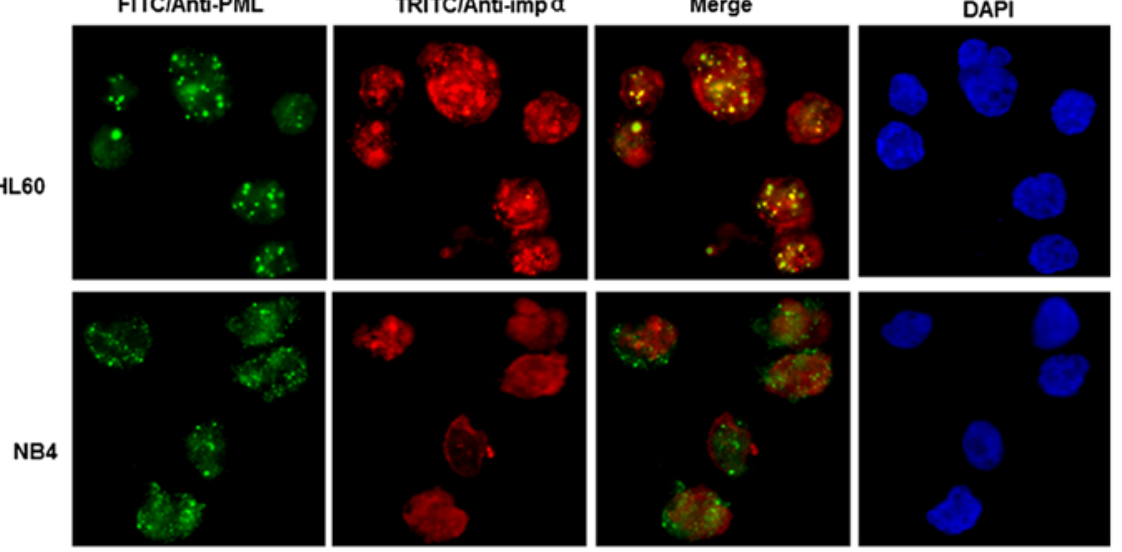

Figure 4. Analyses of PML and importin $\alpha$ co-localization in transiently transfected HEK293 cells and APL cell lines. (A nd B) Localization of PML, PML(NLS $)$, NLS-RAR $\alpha$ and RAR $\alpha$, and their co-localization with importin $\alpha$, in transfected HEK293 cells. (A) Top panel, co-transfection with PCMVHA-PML and PCMV-Myc-importin $\alpha$. Bottom panel, co-transfection with PCMV-HA-PML(NLS-) and PCMV-Myc-importin $\alpha$. (B) Top panel, co-transfection with PCMV-HA-RAR $\alpha$ and PCMV-Myc-importin $\alpha$. Bottom panel, co-transfection with PCMV-HA-NLS-RAR $\alpha$ and PCMV-Myc-importin $\alpha$. Nuclei were visualized by 4',6-diamidino-2-phenylindole staining. An anti-HA antibody was used for HA-tagged proteins (green fluorescence). An anti-Myc antibody was used for Myc-tagged importin $\alpha$ (red fluorescence). (C) Co-localization of PML and importin $\alpha$ in HL60 and NB4 cells. Cells were analyzed by immunofluorescence staining with anti-PML polyclonal (green fluorescence) and anti-importin $\alpha$ monoclonal (red fluorescence) antibodies. Nuclei were visualized by 4',6-diamidino-2-phenylindole staining. Co-localization of PML and importin $\alpha$ is illustrated by yellow fluorescence after overlaying the images.

APL cells. In healthy blood neutrophils, PML showed a clear punctate nuclear pattern of immunostaining (green fluorescence, Fig. 5A and B) that represented its nuclear localization. Importin $\alpha$ immunostaining (red fluorescence, Fig. 5B) also showed a discrete, punctate pattern that was very well co-localized with PML in healthy cells (merged image of yellow fluorescence, Fig. 5B). This result demonstrated excellent co-localization of PML with importin $\alpha$ in healthy cells. However, in primary APL cells, PML exhibited a mostly cytoplasmic localization (Fig. 5A and B). Similarly, importin $\alpha$ had a discrete cytoplasmic localization without co-localization with PML (Fig. 5B). In contrast to primary APL cells, primary M2, M4, M6, and CML leukemia cells had a punctate nuclear pattern of PML expression (Fig. 5A), similar to that in healthy blood neutrophils.

The median age of the APL patients examined in the present study was 30 years (range 14-60) and the study included 11 men and 16 women. Using morphology alone 


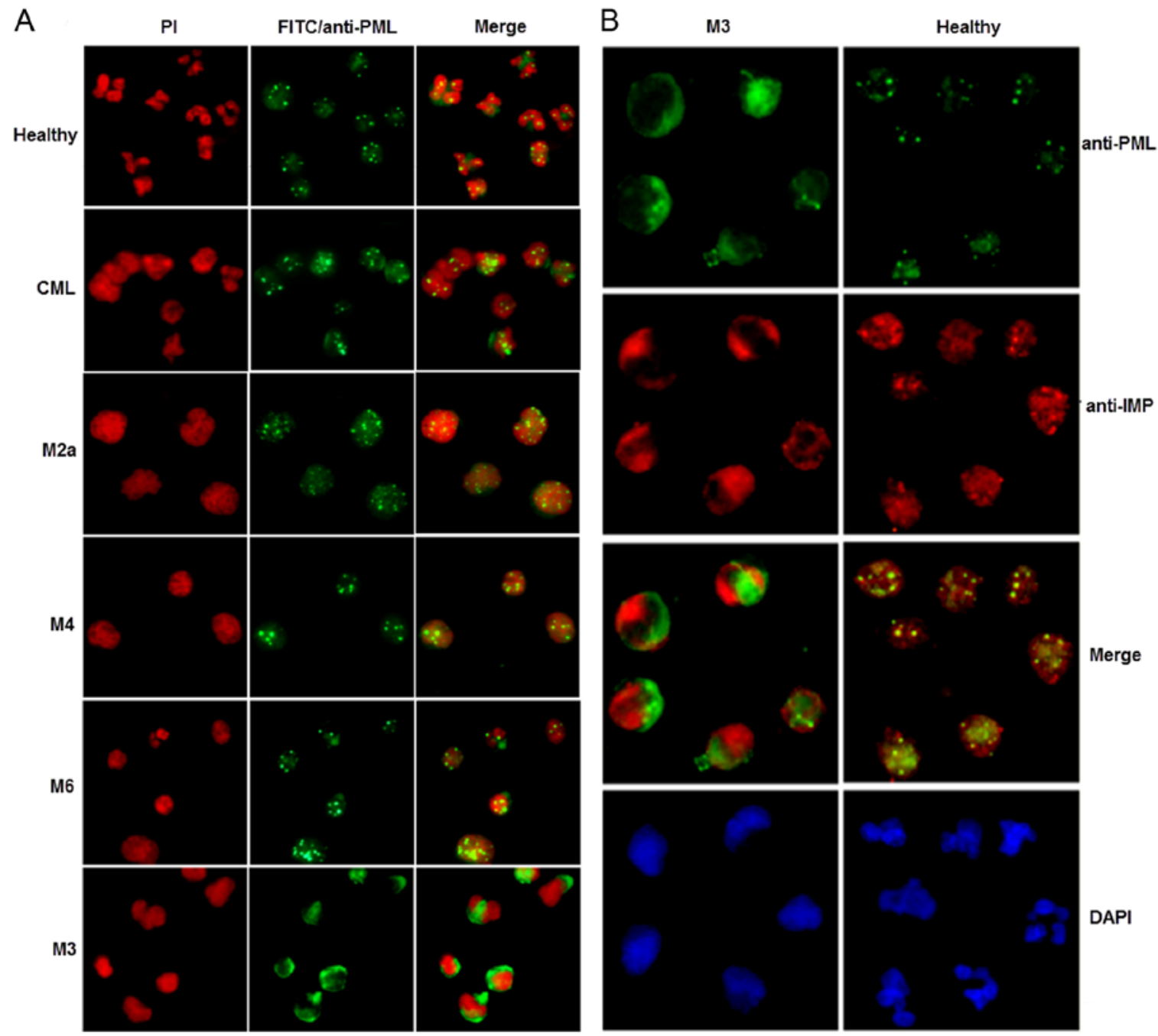

Figure 5. Subcellular localization of PML, and co-localization of PML with importin $\alpha$, in primary human APL cells. (A) Subcellular localization of PML in primary M3, M2a, M4 and M6 AML cells. CML, primary CML cells. Healthy, peripheral blood neutrophils from healthy individuals. Cells were visualized by immunofluorescence microscopy. Propidium iodide was used to stain the nuclei. (B) Co-localization of PML with importin $\alpha$ in primary APL cells Immunofluorescence with rabbit anti-PML, detected with anti-rabbit IgG-fluorescein isothiocyanate (green fluorescence) and mouse anti-importin $\alpha$, detected with anti-mouse IgG-tetramethylrhodamineisothiocyanate (red fluorescence) was used. Co-localization of PML and importin $\alpha$ is illustrated by yellow fluorescence after overlaying the images. M3, primary M3 AML cells. Healthy, peripheral blood neutrophils from healthy patients. Nuclei were visualized by 4',6-diamidino-2-phenylindole staining.

Table II. Comparison of various diagnostic tests for APL.

\begin{tabular}{|c|c|c|c|c|c|c|c|c|}
\hline \multirow[b]{2}{*}{ Test } & \multicolumn{2}{|c|}{$\operatorname{APL}(\mathrm{N}=27)$} & \multicolumn{2}{|c|}{ Non-APL (N=22) } & \multirow[b]{2}{*}{$\begin{array}{c}\text { Sensitivity } \\
(\%)\end{array}$} & \multirow[b]{2}{*}{$\begin{array}{l}\text { Specificity } \\
(\%)\end{array}$} & \multirow[b]{2}{*}{$\begin{array}{l}\text { PPV } \\
(\%)\end{array}$} & \multirow[b]{2}{*}{$\begin{array}{l}\text { NPV } \\
(\%)\end{array}$} \\
\hline & $\begin{array}{c}\text { Correctly identified } \\
(\%)\end{array}$ & $\begin{array}{c}\text { False negative } \\
(\%)\end{array}$ & $\begin{array}{c}\text { Correctly identified } \\
(\%)\end{array}$ & $\begin{array}{c}\text { False negative } \\
(\%)\end{array}$ & & & & \\
\hline Morphology & $23(85.2)$ & $4(14.8)$ & $13(59.1)$ & $9(40.9)$ & 85.1 & 59.1 & 71.9 & 76.5 \\
\hline IF (PML) & 25 (92.6) & $2(7.4)$ & $17(77.3)$ & $5(22.7)$ & 92.6 & 77.3 & 82.8 & 85.0 \\
\hline
\end{tabular}

N, number of patients; APL, acute promyelocytic leukemia; PPV, positive predictive value; NPV, negative predictive value; IF, immunofluorescence; PML, promyelocytic leukemia.

for diagnosis, 23 of 27 APL cases (85.2\%) were correctly identified (Table II). Four cases (14.8\%) were misdiagnosed as either M2 (2 cases) or M4 ( 2 cases). Of the 22 non-APL AMLs, 13 (59.1\%) were categorized correctly by morphology.
However, the possibility of APL could not be ruled out conclusively in $9(40.9 \%)$ cases due to the presence of atypical cells reminiscent of promyelocytes. These 9 cases were finally categorized as M4 (6 cases) or M5 (3 cases). 
Twenty-five of the 27 APL cases (92.6\%) showed an acytoplasmic or microgranular nuclear pattern of immunofluorescence using an anti-PML antibody. Two cases (7.4\%) showed a punctate pattern (false-negative). Seventeen of 22 non-APL AML controls (77.3\%) showed a punctate pattern and $5(22.7 \%)$ showed a microgranular pattern (false-positive) (Table II). Overall, immunofluorescence was significantly superior to morphology alone for the diagnosis of APL $(\mathrm{P}<0.01)$.

\section{Discussion}

In the present study, we showed that a truncated PML protein, PML(NLS), that contained the N-terminal domain of PML without the C-terminal NLS portion, was present in primary APL cells. In contrast, PML(NLS) was not detected in M2 and M4 primary leukemia cells. To determine whether there was sufficient NE activity for this cleavage event, the expression of this enzyme was examined in primary AML cells. Human primary APL cells showed abundant NE activity that was much higher than that in U937 cells. In contrast, neutrophil extracts from normal and NB4 cells did not show PML-RAR $\alpha$ cleavage activity or measurable amounts of NE. Together, these data suggest that PML(NLS) is unique for APL and may be a new diagnostic marker of this disease. PML(NLS) may also play a role in the pathogenesis of APL.

NLS-dependent nuclear protein import is an important regulatory process in the biological information network. Abnormalities or a deficiency of the NLS may disrupt the information network and induce disease. To determine whether a deficiency of the NLS sequence influenced the localization of PML, we compared the subcellular localization of PML and PML(NLS). Immunofluorescence microscopy showed that PML exhibited a nuclear pattern of expression while PML(NLS) aggregates were found in the cytoplasm. In contrast to the micro-punctate nuclear pattern of PML-RARa in control NB4 cells, the PML-RAR $\alpha$ cleavage activity in NB4/p-NE cells yielded a cytoplasmic localization. Similarly, PML exhibited a punctate nuclear pattern in healthy and non-APL cells, while in cells from APL patients there was mostly a cytoplasmic localization pattern of PML(NLS), or a slight micro-punctate nuclear pattern. These data suggest that the NLS plays a key role in the nuclear localization of PML and indicate that analysis of the distribution of PML(NLS') may provide a potential diagnostic method for APL.

Exploring how the NLS mediates the transport of PML into the nucleus, we found that PML and importin $\alpha$ interact in vivo and co-localize in PML NBs. In contrast, HA-PML(NLS ${ }^{-}$) transfected HEK293 and NB4/p-NE cells did not interact with importin $\alpha$. While human primary APL cells exhibit no noticeable co-localization with importin $\alpha$ in the nucleus, non-APL AML cells and neutrophils from healthy cells containing wild-type PML protein, exhibit obvious co-localization. These data suggest that PML is transported into the nucleus through its NLS sequence and interacts with importin $\alpha$. The interaction between PML and importin $\alpha$ could also be regarded as a diagnostic label to distinguish APL and non-APL leukemia.

APL is a highly aggressive disease that requires prompt diagnosis and specific early intervention. PML-RAR $\alpha$-positive
APL patients often present with coagulopathy. Without treatment, $\sim 40 \%$ of the patients develop pulmonary and cerebral hemorrhages that can be lethal (26). The latest research on methods for detecting PML-RAR $\alpha$ rearrangements, including a flow cytometric immunobead assay (27), reverse transcription polymerase chain reaction (RT-PCR) (28), and a new high-speed droplet RT-PCR (29), has focused on improved detection of the PML-RAR $\alpha$ fusion gene and protein rather than exploring new indicators. As the current diagnostic gold standard, RT-PCR for PML-RAR $\alpha$ is time-consuming. There is thus a need for a rapid and accurate diagnostic test for APL.

Since early promyelocytes produce massive amounts of NE, cleavage events occur at an early stage of APL. Consequently, the immunofluorescence assay for assessing the distribution of PML(NLS') enables an earlier diagnosis of APL and allows clinicians to begin treatment sooner. However, this immunofluorescence assay cannot be used to detect variants of APL that involve the formation of fusion genes with RAR $\alpha$, or genes other than PML [e.g. promyelocytic zinc finger; $t(11 ; 17)$ and nucleophosmin; $\mathrm{t}(5 ; 17)]$. To detect PML proteins in cell lysates, antibodies were raised against the $\mathrm{N}$-terminal domain of PML, upstream of the BCR3 break point region. This domain is present in all known PML-RAR $\alpha$ fusion proteins. Thus, these anti-PML antibodies recognize PML and PML-RAR $\alpha$, as well as PML(NLS). We are committed to developing a new antiPML antibody that is directed against the NLS domain of PML and is able to distinguish between PML and PML(NLS). Such an antibody may be useful clinically for the diagnosis of APL.

In conclusion, the present study describes a simple, fast and accurate immunofluorescence assay for the detection of PML(NLS) localization that can be used to diagnose APL. Compared with the traditional morphologic test, sensitivity was increased by nearly $7.5 \%$ and specificity increased from 59.1 to $77.3 \%$. These results indicate that immunofluorescence has significant potential for detecting PML(NLS) and may be useful in diagnosing APL. Two false negatives were found among 27 cases of APL (7.4\%). Possible causes of these false negatives include mutations other than $\mathrm{t}(15 ; 17)$, insufficient material on the slides, or an artifact of prolonged storage (30). All cases were $t(15 ; 17)$-positive and stained better immediately rather than following storage. Thus, false negatives with stored samples may be a limitation of the test.

Overall, the data reported in the present study strongly suggest that the NLS plays a critical role in the nuclear localization of PML by interacting with importin $\alpha$. NE-mediated cleavage leading to the formation of PML(NLS) was unique in APL patients compared with other non-APL leukemias, suggesting that PML(NLS) may represent a novel biomarker for APL. The detection of cytoplasmic PML(NLS) in APL patients by immunofluorescence staining could be an early diagnostic method for APL.

\section{Acknowledgements}

The present study was supported by the National Natural Science Foundation of China (no. 81171658) and the Natural Science Foundation Project of CQ CSTC (no. 2011BA5037). We kindly thank Dr Jianbin Chen of The First Affiliated Hospital of Chongqing Medical University, for his kind contribution of patient samples to the present study. 


\section{References}

1. Puccetti E and Ruthardt M: Acute promyelocytic leukemia: PML/RARalpha and the leukemic stem cell. Leukemia 18: 1169-1175, 2004.

2. Wang K, Wang P, Shi J, Zhu X, He M, Jia X, Yang X, Qiu F, Jin W, Qian M, et al: PML/RARalpha targets promoter regions containing PU.1 consensus and RARE half sites in acute promyelocytic leukemia. Cancer Cell 17: 186-197, 2010.

3. Grisolano JL, Wesselschmidt RL, Pelicci PG and Ley TJ: Altered myeloid development and acute leukemia in transgenic mice expressing PML-RAR alpha under control of cathepsin G regulatory sequences. Blood 89: 376-387, 1997.

4. He LZ, Tribioli C, Rivi R, Peruzzi D, Pelicci PG, Soares V, Cattoretti G and Pandolfi PP: Acute leukemia with promyelocytic features in PML/RARalpha transgenic mice. Proc Natl Acad Sci USA 94: 5302-5307, 1997.

5. Westervelt P, Lane AA, Pollock JL, Oldfather K, Holt MS, Zimonjic DB, Popescu NC, DiPersio JF and Ley TJ High-penetrance mouse model of acute promyelocytic leukemia with very low levels of PML-RARalpha expression. Blood 102: $1857-1865,2003$

6. Early E, Moore MA, Kakizuka A, Nason-Burchenal K, Martin P, Evans RM and Dmitrovsky E: Transgenic expression of PML/RARalpha impairs myelopoiesis. Proc Natl Acad Sci USA 93: 7900-7904, 1996.

7. Lane AA and Ley TJ: Neutrophil elastase cleaves PML-RARalpha and is important for the development of acute promyelocytic leukemia in mice. Cell 115: 305-318, 2003

8. Lane AA and Ley TJ: Neutrophil elastase is important for PML-retinoic acid receptor alpha activities in early myeloid cells. Mol Cell Biol 25: 23-33, 2005.

9. Gao YM, Zhong L, Zhang X, Hu XX and Liu BZ: PML(NLS') inhibits cell apoptosis and promotes proliferation in HL-60 cells. Int J Med Sci 10: 498-507, 2013.

10. Hu XX, Zhong L, Zhang X, Gao YM and Liu BZ: NLS-RARo promotes proliferation and inhibits differentiation in HL-60 cells. Int J Med Sci 11: 247-254, 2014

11. Sardiello M, Cairo S, Fontanella B, Ballabio A and Meroni G: Genomic analysis of the TRIM family reveals two groups of genes with distinct evolutionary properties. BMC Evol Biol 8 : 225, 2008.

12. Salomoni P, Ferguson BJ, Wyllie AH and Rich T: New insights into the role of PML in tumour suppression. Cell Res 18: 622-640, 2008.

13. Geng Y, Monajembashi S, Shao A, Cui D, He W, Chen Z, Hemmerich $\mathrm{P}$ and Tang J: Contribution of the C-terminal regions of promyelocytic leukemia protein (PML) isoforms II and V to PML nuclear body formation. J Biol Chem 287: 30729-30742, 2012.

14. Guan D and Kao HY: The function, regulation and therapeutic implications of the tumor suppressor protein, PML. Cell Biosci 5: 60, 2015.

15. Ding W, Tong Y, Zhang X, Pan M and Chen S: Study of arsenic sulfide in solid tumor cells reveals regulation of nuclear factors of activated T-cells by PML and p53. Sci Rep 6: 19793, 2016.

16. Fu C, Ahmed K, Ding H, Ding X, Lan J, Yang Z, Miao Y, Zhu Y, Shi Y, Zhu J, et al: Stabilization of PML nuclear localization by conjugation and oligomerization of SUMO-3. Oncogene 24 : $5401-5413,2005$.

17. Rabellino A, Carter B, Konstantinidou G, Wu SY, Rimessi A, Byers LA, Heymach JV, Girard L, Chiang CM, Teruya-Feldstein J, et al: The SUMO E3-ligase PIAS1 regulates the tumor suppressor PML and its oncogenic counterpart PML-RARA. Cancer Res 72: 2275-2284, 2012.
18. Weisshaar SR, Keusekotten K, Krause A, Horst C, Springer HM, Göttsche K, Dohmen RJ and Praefcke GJ: Arsenic trioxide stimulates SUMO-2/3 modification leading to RNF4-dependent proteolytic targeting of PML. FEBS Lett 582: 3174-3178, 2008.

19. Jin G, Gao Y and Lin HK: Cytoplasmic PML: From molecular regulation to biological functions. J Cell Biochem 115: 812-818, 2014.

20. Lange A, Mills RE, Lange CJ, Stewart M, Devine SE and Corbett AH: Classical nuclear localization signals: Definition, function, and interaction with importin alpha. J Biol Chem 282: 5101-5105, 2007.

21. Xiao C, Zhong L, Shan Z, Xu T, Gan L, Song H, Yang R, Li L and Liu B: NLS-RAR $\alpha$ inhibits the effects of all-trans retinoic acid on NB4 cells by interacting with P38 $\alpha$ MAPK. Int J Med Sci 13: 611-619, 2016.

22. FanelliM,MinucciS, GelmettiV,NerviC,Gambacorti-PasseriniC and Pelicci PG: Constitutive degradation of PML/RARalpha through the proteasome pathway mediates retinoic acid resistance. Blood 93: 1477-1481, 1999.

23. Jing Y, Xia L, Lu M and Waxman S: The cleavage product deltaPML-RARalpha contributes to all-trans retinoic acidmediated differentiation in acute promyelocytic leukemia cells. Oncogene 22: 4083-4091, 2003.

24. Le XF, Yang P and Chang KS: Analysis of the growth and transformation suppressor domains of promyelocytic leukemia gene, PML. J Biol Chem 271: 130-135, 1996.

25. Gallagher R, Collins S, Trujillo J, McCredie K, Ahearn M, Tsai S, Metzgar R, Aulakh G, Ting R, Ruscetti F, et al: Characterization of the continuous, differentiating myeloid cell line (HL-60) from a patient with acute promyelocytic leukemia. Blood 54: 713-733, 1979.

26. Taube F, Stölzel F, Thiede C, Ehninger G, Laniado M and Schaich M: Increased incidence of central nervous system hemorrhages in patients with secondary acute promyelocytic leukemia after treatment of multiple sclerosis with mitoxantrone? Haematologica 96: e31, 2011.

27. Dekking EH, van der Velden VH, Varro R, Wai H, Böttcher $S$, Kneba M, Sonneveld E, Koning A, Boeckx N, Van Poecke N, et al; EuroFlow Consortium (EU-FP6, LSHB-CT-2006-018708): Flow cytometric immunobead assay for fast and easy detection of PML-RARA fusion proteins for the diagnosis of acute promyelocytic leukemia. Leukemia 26: 1976-1985, 2012.

28. Dolz S, Barragán E, Fuster O, Llop M, Cervera J, Such E, De Juan I, Palanca S, Murria R, Bolufer P, et al: Novel real-time polymerase chain reaction assay for simultaneous detection of recurrent fusion genes in acute myeloid leukemia. J Mol Diagn 15: 678-686, 2013.

29. Sueki A, Matsuda K, Taira C, Yamaguchi A, Koeda H, Takagi F, Kobayashi Y, Sugano $\mathrm{M}$ and Honda T: Rapid detection of $P M L-R A R A$ fusion gene by novel high-speed droplet-reverse transcriptase-polymerase chain reaction: Possibility for molecular diagnosis without lagging behind the morphological analyses. Clin Chim Acta 415: 276-278, 2013.

30. Gomis F, Sanz J, Sempere A, Plumé G, Senent ML, Pérez ML, Cervera J, Moscardó F, Bolufer P, Barragán E, et al: Immunofluorescent analysis with the anti-PML monoclonal antibody PG-M3 for rapid and accurate genetic diagnosis of acute promyelocytic leukemia. Ann Hematol 83: 687-690, 2004. 